Journal of Physics and Its Applications

Journal homepage : https://ejournal2.undip.ac.id/index.php/jpa/index

\title{
Mapping various cooking oil using fluorescence polarization
}

\author{
K. Sofjan Firdausi ${ }^{1}$, I. Afiefah ${ }^{2}$, H. Sugito ${ }^{3}$, and Much. Azam ${ }^{4}$ \\ 1Department of Physics, Diponegoro University; firdausi@fisika.undip.ac.id \\ 2 Physics Undergraduate Study Program, Department of Physics, Diponegoro University; izzahafiefah@st.fisika.undip.ac.id \\ 3 Department of Physics, Diponegoro University; herinuha@gmail.com \\ ${ }_{4}$ Department of Physics, Diponegoro University; azamfisika@gmail.com
}

\section{A R T I C L E I N F O}

\section{Article history:}

Received : September 2018

Accepted : November 2018

Available online : November 2018

\section{Keywords:}

Mapping Cooking oils

Critical polarizer's angle

Fluorescence polarization

Change of polarization angle

\section{Introduction}

Fluorescence polarization of light is simply defined as a change of polarization angle of incoming light due to fluorescence scattering. The change of light polarization through fluorescence method in sample of cooking oils has been very interesting and considered as new finding in our works [1, 2], because we have used direct measurement of change of polarization angle between fluorescence light and the incoming light for determination of cooking oil quality and it has provided more significant results than standard methods. The direct measurement of change polarization angle is an unusual method for cooking oil quality testing due to very small polarization. However this problem can be overcome by repetition of measurement in several times to reduce error or uncertainties in parallax observation. The direct measurement of fluorescence polarization angle is simpler than other sophisticated standard methods. Usually, in case of fluorescence methods, the quality of cooking oils and their types are determined and measured using fluorescence spectroscopy methods with additional instruments [3-5] or additional assistant methods. Other method, as an example, for comprehensive investigation of halal food due to lard contamination, an expensive sophisticated GCMS and its derivative instrument are to be used [6].

In this paper we use direct measurement of polarization angle of light to observe various types of cooking oils. The difference of our method with fluorescence spectroscopy or GCMS method is very clear, that we use simply direct measurement of polarization angle. The new finding of our previous reports shows that the symmetry polarization between polarizer's angle $\varphi$ from $0^{\circ}-90^{\circ}$ and from $90^{\circ}-180^{\circ}$ gives interesting results such as characteristics values of $\theta$ at $\varphi=0^{\circ}$, at $\varphi=90^{\circ}$, critical value of $\varphi_{c}$ where the polarization $\theta$ tends to zeroand also the average value of $\theta_{A v g}$ [1]. From our previous works[1, 2], the obtained data and its expression of the results are very possible to be developed for various types of oils, their quality and halal cases, and therefore it is still to be improved. The comprehensive physical explanation of the existence for critical value $\varphi_{c}$ is still interesting to be discussed. In this report, the data of various cooking oils will be expressed through the change of fluorescence polarization $\theta$ against the polarization of the incoming light or polarizer's angle $\varphi$. From critical value $\varphi_{c}$, the cooking oils will be grouped and the best possible mapping various oil will be discussed. 


\section{Methods}

The sample was various cooking oils presented in the table 1 below.

Table 1: ${ }^{a}$ Various cooking oils examined in the research

\begin{tabular}{lll}
\hline Cooking oil & code & Date of measurement \\
\hline VCO & $*$ & \\
Olive & $\diamond$ & \\
Soybean & $\square$ & \\
Corn & $\Delta$ & \\
Palm & $\times$ & 24 October 2017 \\
Chicken1 & +1 & \\
Chicken2 & +2 & \\
Chicken3 & +3 & \\
Lard1 & $\mathrm{o} 1$ & \\
Lard2 & $\mathrm{o} 2$ & \\
Lard3 & $\mathrm{o3}$ & \\
\hline Canola & $-\mathrm{C}$ & \\
Chicken1 & $+1^{*}$ & \\
Chicken2 & $+2^{*}$ & \\
Chicken3 & $+3^{*}$ & 19 September 2017 \\
Lard1 & $\mathrm{o} 1^{*}$ & \\
Lard2 & $\mathrm{o} 2^{*}$ & \\
Lard3 & $\mathrm{o} 3^{*}$ & \\
\hline Olive & $\diamond^{*}$ & \\
Soybean & $\square *$ & \\
Corn & $\Delta^{*}$ & \\
Palm & $\times^{*}$ & Aug-Oct 2018 \\
Rice bran & $-\mathrm{R}$ & \\
Sunflower & $-\mathrm{S}$ & \\
Chicken4 & +4 & \\
Lard4 & $\mathrm{o} 4$ & \\
Lard5 & $\mathrm{o5}$ & \\
\hline the data acquistion for 0 live, soybean corn, and &
\end{tabular}

$a$ the data acquisition for olive, soybean, corn, and palm was taken twice in 24 Oct 2017 and Aug-Oct 2018.

Chicken oil 1, 2, 3 and lard 1, 2, 3 were taken twice in 24 Oct 2017 and 19 Sep 2017.

The basic data acquisition of the change of fluorescence polarization angle was referred to Firdausi et al [7]. To ensure the symmetry characteristics polarization angle, a measurement of scattering polarization of water molecules was used to calibrate the whole system of the instrument.

\section{Results and Discussions}

Fig. 1 shows profile $\theta$ vs. $\varphi$ of pure scattering polarization angle on water and we neglected absorption and Raman scattering due to verysmall size of water molecules relative to oil molecules.

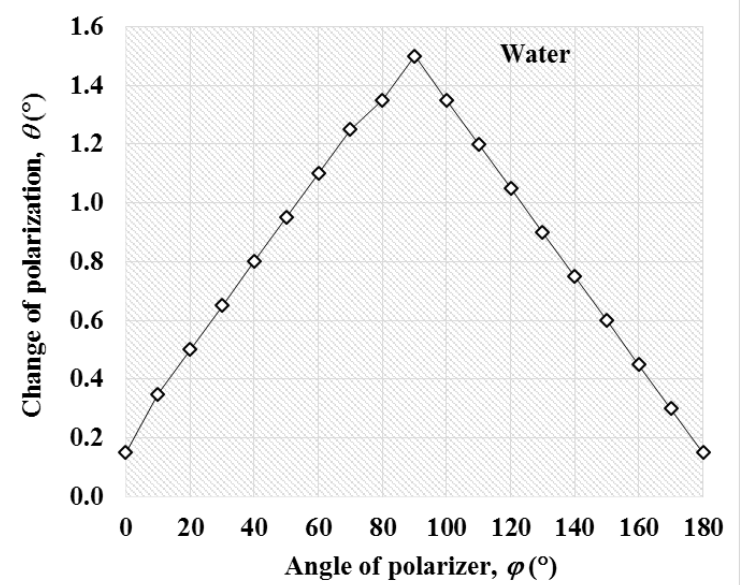

Fig. 1: Polarization angle $\theta$ vs. $\varphi$ of scattering on water The symmetric value $\theta$ as polarizer's angle $\varphi$ goes from $0^{\circ}$ to $90^{\circ}$ and from $90^{\circ}$ to $180^{\circ}$ is considered sufficient that the system of instrument was well calibrated.

For the cooking oils, the profiles of fluorescence polarization angle $\theta$ vs. $\varphi$ for all samples are symmetry at polarizer's anglefrom $\varphi=0^{\circ}$ to $180^{\circ}$, however each sample shows unique characteristics. Here, the profile of some examples (not all samples are shown) taken in Aug-Oct 2018 is shown in fig 2.

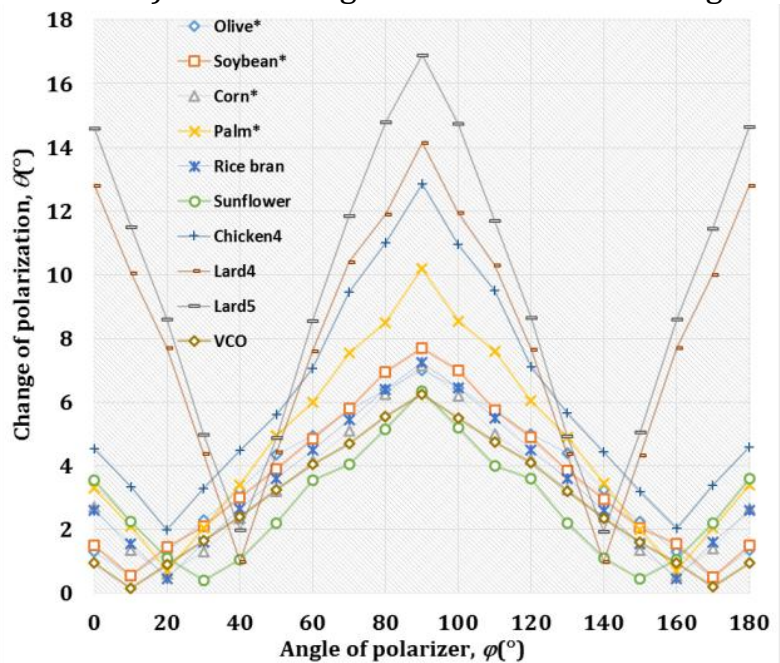

Fig. 2: Polarization angle $\theta$ vs. $\varphi$ for samples taken in AugOct 2018

Since the data is symmetry from $\varphi=0^{\circ}$ to $180^{\circ}$, we discuss only the unique values for each sample from $\varphi=0^{\circ}$ to $90^{\circ}$ only. The minimum value of fluorescence polarization angle $\theta_{\text {min }}$ for each cooking oil results at different or unique polarizer's angle so called critical polarizer's angle $\varphi_{\text {c }}$. Comparing the minimum pure scattering angle of water at $\varphi_{\mathrm{c}}=0^{\circ}$ (fig.1) to fluorescence scattering angle of oil at $\varphi_{c} \geq$ $10^{\circ}$ (fig.2), the possible physical explanation should be placed at characteristics molecules and different transition and emission of the different wavelength of fluorescence light from each most fatty acids composition for each oil. Since the molecules of cooking oil are in average much greater than water molecules, a complex combination of pure scattering and fluorescence scattering is taken place in oil and results in different $\varphi_{\mathrm{c}}$ and much high value $\theta$ in comparison to the pure scattering on water molecules. It is still an interesting task to answer questions, why $\varphi_{\mathrm{c}}$ for certain oil increases a different value in step $10^{\circ}, 20^{\circ}, 30^{\circ}$ and $40^{\circ}$ or why for lard is the greatest at $40^{\circ}$. Because the direct measurement is the change of polarization angle, the answers of the question should be based on the asymmetrical or symmetrical triglyceride molecules, the orientation of molecules, size of triglyceride molecules in oil, and characteristics of transition on energy levels in each sample. It should be comprehensively investigated through the role of the pattern of molecule orientation, transition level and the data of spectroscopy.

There are at least four characteristics values that can be noticed i.e. critical value $\varphi_{\text {c }}$,polarization angle $\theta_{0}\left(\right.$ at $\varphi=0^{\circ}$ ), peak polarization angle $\theta_{\mathrm{P}}$ (at $\varphi=90$ ), 
and minimum polarization angle $\theta_{\text {min }}$ in which $\varphi_{\mathrm{c}}$ takes place. In our previous reports, we have used average polarization angle $\theta_{\text {Avg }}$ vs. critical value $\varphi_{c}$ and it has shown an interesting map of cooking oils, but the samples were only canola oil, chicken oil, and lard [1]. In this report, although some oils can be mapped and distinguished significantly, however some others show similar results with each other such as olive and soybean that have the same $\varphi_{\mathrm{c}}=$ $10^{\circ}$, and Corn, palm and chicken clearly have the same $\varphi_{\mathrm{c}}=20^{\circ}$. Therefore to differentiate among them it should be modified using other unique values. More significant result shows that lard has greatest value $\varphi_{\mathrm{c}}=40^{\circ}$. It is discriminated from other oils and it provides such as an example of a determination for lard contamination in the future. Table 2 shows the values $\varphi_{\mathrm{c}}, \theta_{\mathrm{min}}, \theta_{0}$, and $\theta_{\mathrm{p}}$ for all samples.

Table 2: The values $\varphi_{\mathrm{c}}, \theta_{\min }, \theta_{0}$, and $\theta_{\mathrm{p}}$ for all samples

\begin{tabular}{|c|c|c|c|c|c|}
\hline Sample & Code & $\varphi_{c}\left({ }^{\circ}\right)$ & $\theta_{\min }\left({ }^{\circ}\right)^{b}$ & $\theta_{0}\left({ }^{\circ}\right)$ & $\theta_{\mathrm{p}}\left({ }^{\circ}\right)$ \\
\hline VCO & $*$ & 10 & 0.15 & 0.95 & 6.25 \\
\hline Olive & $\Delta$ & 10 & 0.30 & 1.05 & 6.90 \\
\hline Soybean & $\square$ & 10 & -0.90 & 1.80 & 7.35 \\
\hline Corn & $\Delta$ & 20 & -0.40 & 2.55 & 6.95 \\
\hline Palm & $x$ & 20 & -0.35 & 3.10 & 9.70 \\
\hline Chicken1 & +1 & 20 & -2.15 & 9.05 & 27.55 \\
\hline Chicken2 & +2 & 20 & -3.45 & 9.40 & 23.45 \\
\hline Chicken3 & +3 & 20 & -2.10 & 10.15 & 29.10 \\
\hline Lard1 & 01 & 40 & -2.10 & 15.85 & 18.15 \\
\hline Lard2 & 02 & 40 & -1.85 & 14.80 & 17.00 \\
\hline Lard3 & 03 & 40 & 1.00 & 11.50 & 13.75 \\
\hline Canola & $-\mathrm{C}$ & 30 & -0.30 & 3.05 & 6.00 \\
\hline Chicken1 & $+1^{*}$ & 20 & -1.25 & 9.70 & 26.15 \\
\hline Chicken2 & $+2^{*}$ & 20 & -3.20 & 9.10 & 23.15 \\
\hline Chicken3 & $+3^{*}$ & 20 & -1.65 & 10.10 & 27.05 \\
\hline Lard1 & $01^{*}$ & 40 & -1.80 & 15.60 & 17.95 \\
\hline Lard2 & o2* & 40 & -1.60 & 14.20 & 16.00 \\
\hline Lard3 & o3* & 40 & -0.80 & 11.60 & 13.55 \\
\hline Olive* & $\diamond^{*}$ & 10 & 0.55 & 1.30 & 7.00 \\
\hline Soybean* & $\square *$ & 10 & -0.55 & 1.50 & 7.70 \\
\hline Corn* & $\Delta^{*}$ & 20 & -0.60 & 2.70 & 7.15 \\
\hline Palm* & $x^{*}$ & 20 & -0.70 & 3.30 & 10.20 \\
\hline Rice bran & $-\mathrm{R}$ & 20 & -0.45 & 2.60 & 7.25 \\
\hline Sunflower & $-S$ & 30 & -0.40 & 3.55 & 6.35 \\
\hline Chicken4 & +4 & 20 & -2.00 & 4.55 & 12.85 \\
\hline Lard4 & 04 & 40 & 1.00 & 12.8 & 14.15 \\
\hline Lard5 & 05 & 40 & -2.05 & 14.60 & 16.90 \\
\hline
\end{tabular}

$b$ the minus "-" sign for $\theta_{\min }$ indicates that the direction of polarization angle in the left at critical value $\varphi_{c}$

All uncertainties of values $\theta$ from measurement was obtained approximately $0.03^{\circ}$ for all samples and it is considerably accurate. All samples are almost can be differentiated. The exception is at $\varphi_{\mathrm{c}}=10^{\circ}$ for VCO $\left(\theta_{0}=0.95^{\circ}\right)$ vs. Olive $\left(\theta_{0}=1.05^{\circ}\right)$, and at $\varphi_{\mathrm{c}}=20^{\circ}$ for corn $\left(\theta_{0}=2.55^{\circ}\right)$ vs. Rice bran $\left(\theta_{0}=2.60^{\circ}\right)$, which is very small difference and will be seen overlapped each other in the map. This overlapping is considerably understandable because VCO and Olive are relatively very good oil quality. The similarity of VCO and Olive at $\varphi_{\mathrm{c}}$ and $\theta_{0}$ is not situated at similarity of the composition, which is actually very different in composition. The composition in VCO shows medium chain of saturated fatty acid molecules that yields in to smallest homogeneous asymmetry and symmetry of triglyceride molecules from others and results to smallest $\theta_{0}$. The olive oil shows usually highest monounsaturated fatty acids that yields in to highest symmetrical triglyceride molecules and results to small $\theta_{0}$, as well. Meanwhile, the similarity between corn (saturated fat 25\%, mono-saturated fat 38\%, poly-unsaturated fat 37\%) and rice bran (saturated fat 19\%, mono-saturated fat $28 \%$, poly-unsaturated fat $53 \%$ ) is almost equal for three highest composition i.e. saturated, monounsaturated, and poly-unsaturated fatty acids that leads to the same proportional asymmetrical triglyceride molecules and results to the same relative intermediate change of polarization.

First, for mapping, we improve the previous results that only used $\theta_{\mathrm{AvgVS}} \varphi_{\mathrm{c}}[1]$, and propose now to use $\theta_{0}$ and $\theta_{\mathrm{p}}$ instead of $\theta_{\mathrm{Avg}}$. From table 2 there are some significant figures that can be exploited, because the critical polarizer's angle is unique for certain cooking oils. The value $\theta_{0}$ and $\theta_{\text {pare also }}$ different among the sample. Definitely, $\theta_{0}$ and $\theta_{\text {pare }}$ relative smaller for vegetable oils (olive, soybean, corn, palm, and canola) than animal oils (chicken oil and lard). Therefore, to map each sample, we tried to choose two models of map which each consists of axis $\theta_{0}$ vs. $\varphi_{\text {c }}$ and axis $\theta_{\mathrm{p}}$ vs. $\varphi_{\mathrm{c}}$. Fig. 3 and fig. 4 show a model of map using axis $\theta_{0}$ vs. $\varphi_{\mathrm{c}}$ and axis $\theta_{\mathrm{p}}$ vs. $\varphi_{\mathrm{c}}$, respectively.

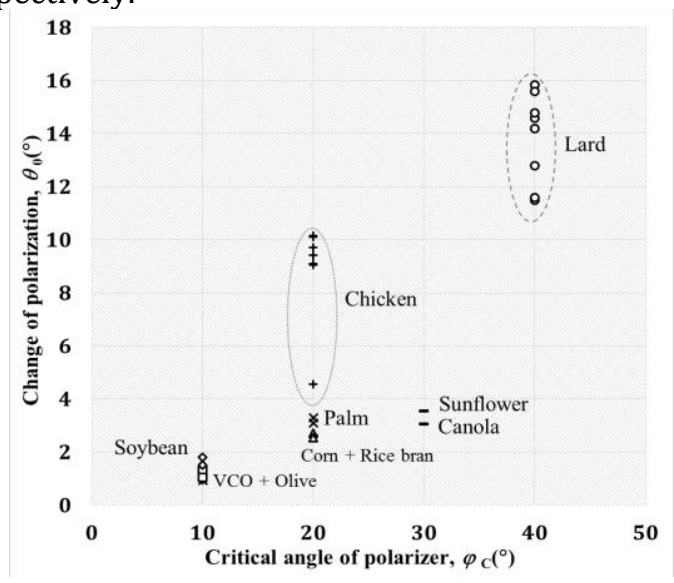

Fig. 3: Mapping sample using axis $\theta_{0}$ vs. $\varphi_{\mathrm{c}}$

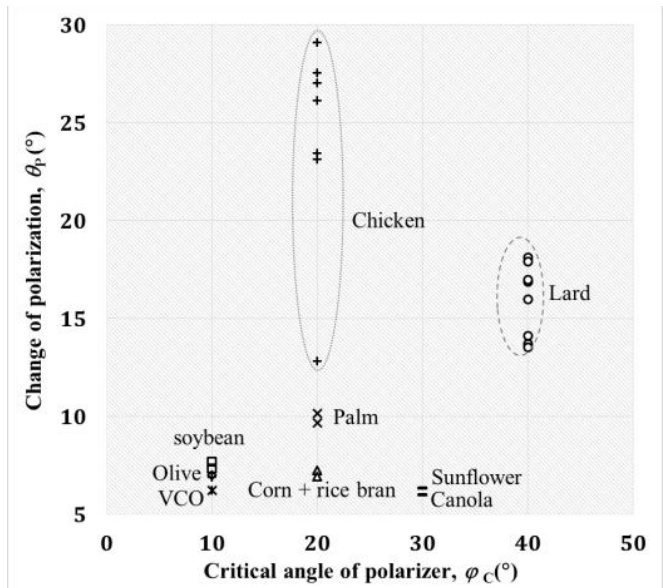

Fig. 4: Mapping sample using axis $\theta$ vs. $\varphi_{\mathrm{c}}$

Relative to the range $\theta_{0}$ from $0^{\circ}$ to $16^{\circ}$ (fig. 3 ) and the range $\theta_{\mathrm{P}}$ from $5^{\circ}$ to $30^{\circ}$ (fig. 4 ), these values $\theta_{0}$ and $\theta_{\mathrm{P}}$ for pair of olive-olive*, soybean-soybean*, corn- 
corn*, and palm-palm* show are not significantly difference, showing that there were no relative change of oil quality in two different time of measurement. Both of fig. 3 and fig.4 are able to display several discriminant values among the cooking oils, although some different oils such as olive and soybean seem overlap each other due to the same $\varphi_{\mathrm{c}}=10^{\circ}$ and slightly different values of $\theta_{0}$ and $\theta_{\mathrm{p}}$ in the vertical axis. The critical value $\varphi_{\mathrm{c}}=30^{\circ}$ and the lowest value $\theta_{\mathrm{p}}$ for canola and sunflower provide a most suitable place and a most unique map for this oil from others as seen in fig.4. Corn, palm, and chicken oil although have the same $\varphi_{\mathrm{c}}=$ $20^{\circ}$ however can be distinguished each other due to significant different value $\theta_{\mathrm{p}}$. Particular consideration of chicken oil can be seen that it has very high value $\theta_{0}$ and $\theta_{\mathrm{P}}$ in comparison to vegetable oils. The most important information is provided by lard which shows very unique $\varphi_{\mathrm{c}}=40^{\circ}, \theta_{0}$ and $\theta_{\mathrm{P}}$. The highest $\varphi_{\mathrm{c}}$ for lard among the cooking oils has already differentiated significant characteristics. In addition, the high $\theta_{0}$ and $\theta_{\mathrm{p}}$ relative to the vegetable oils can be conducted to the halal examination or testing lard contamination. The lack of mapping in fig. 3 and fig. 4 is still the overlapping values of olive + soybean and corn + rice bran oil at the whole range $\theta_{0}$ from $0^{\circ}$ to $16^{\circ}$ (fig. 3 ) and the range $\theta_{\mathrm{p}}$ from $5^{\circ}$ to $30^{\circ}$ (fig.4). To obtain the finest resolution,other modified mapping can be proposed using subtraction of value $\Delta \theta_{-}=\theta_{\mathrm{p}}-\theta_{0}$ vs. $\varphi_{\mathrm{c}}$ and addition of value $\Delta \theta_{+}=\theta_{0}+\theta_{\mathrm{p}}$ vs. $\varphi_{\mathrm{c}}$ as seen in fig.5 and fig.6.

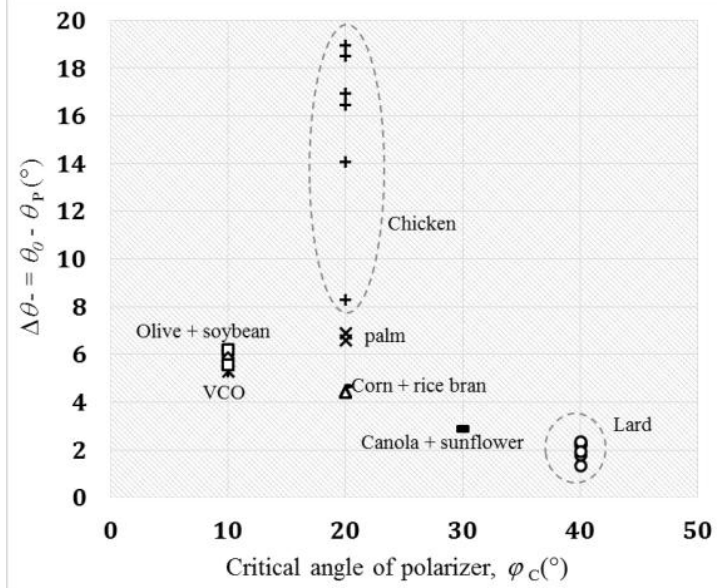

Fig. 5: Modified mapping sample using axis $\Delta \theta$ - vs. $\varphi_{\mathrm{c}}$

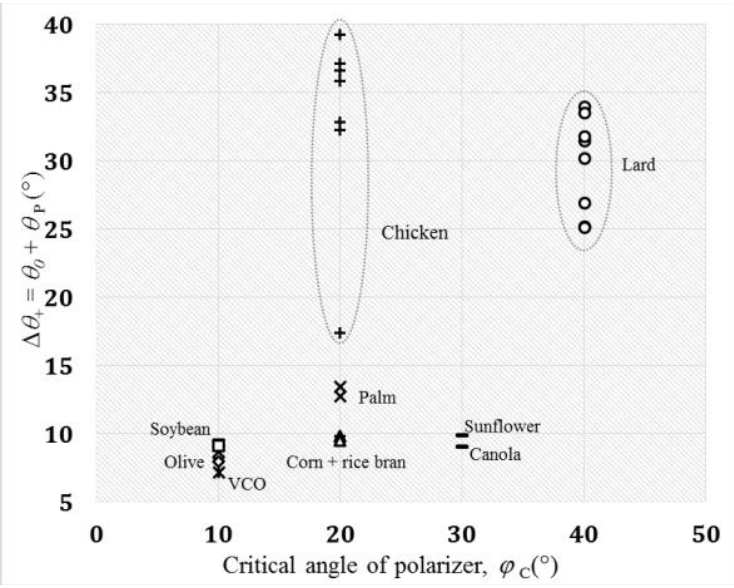

Fig. 6: Modified mapping sample using axis $\Delta \theta_{+}$vs. $\varphi_{\mathrm{c}}$
The modified map using subtraction of value $\Delta \theta_{-}=$ $\theta_{\mathrm{p}}-\theta_{0}$ vs. $\varphi_{\mathrm{c}}$ gives only smallest value $\Delta \theta_{-}$of lard (fig.5) and still provides halal test of cooking oil in the next research. The lack remains by overlapping olive + soybean oil and corn + rice bran. And in other hand, the modified map using addition of value $\Delta \theta_{+}=$ $\theta_{0}+\theta_{\mathrm{p}}$ vs. $\varphi_{\mathrm{c}}$ (fig.6) just looks like mapping sample using $\theta_{\mathrm{P}}$ vs. $\varphi_{\mathrm{c}}$ (fig.4) and still the discrimination between olive and soybean, and alsothe discrimination between corn and rice bran oil are to be increased. Therefore, for mapping various cooking oils should be restricted by range of vegetable only or by range of animal oils only. To differentiate chicken oil from lard, it is relatively simple because of the significantly different values $\varphi_{\mathrm{c}}$, i.e. $\varphi_{\mathrm{c}}=20^{\circ}$ for chicken and $\varphi_{\mathrm{c}}=40^{\circ}$ for lard. It seems also that for mapping lard contamination, all models of the map in fig.3, fig.4, fig. 5 and fig. 6 give a good opportunity due to highest value $\varphi_{\mathrm{c}}=40^{\circ}$. The model for mapping vegetable oils only seems operative if it is used narrow range of $\theta$ as shown in fig.7 using axis $\Delta \theta_{+}$vs. $\varphi_{\text {c. }}$

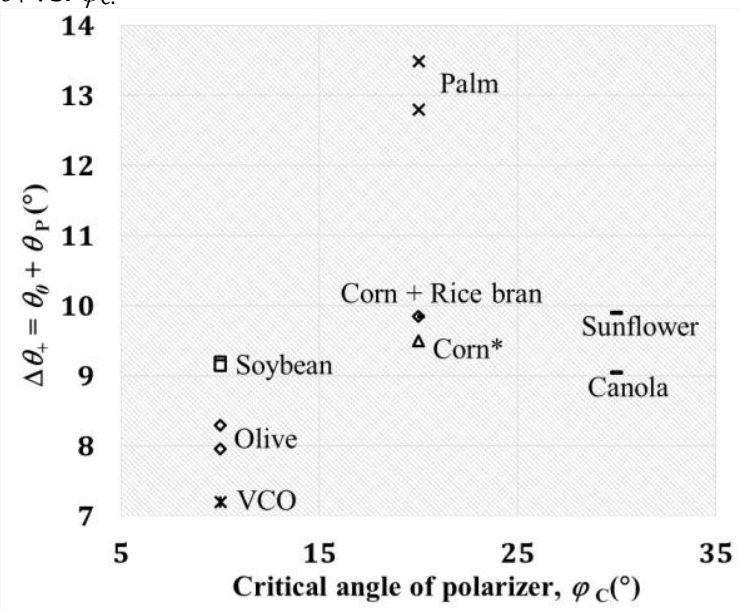

Fig. 7: Modifiedmapping sample using axis $\Delta \theta_{+}$vs. $\varphi_{\mathrm{c}}$ for vegetable oils

The corn and rice bran could be overlapped as seen in fig.7 due to similar distribution of fatty acids. Palm oil has highest composition in saturated $(>50 \%)$ and mono-unsaturated (>30\%) fatty acids that could lead high value $\theta$. Sunflower and canola have the same $\varphi_{c}=30^{\circ}$ but their distribution of highest composition of fatty acids is different. Sunflower has two highest composition in mono- and polyunsaturated fatty acids that can result asymmetry and symmetry of triglycerides in proportional number, while almost more $80 \%$ of monounsaturated fatty acids in canola could combine in to symmetrical triglycerides that results smaller $\theta$ than in sunflower. The model of map using axis $\Delta \theta_{+}$vs. $\varphi_{c}$ seems more advantage than using axis $\Delta \theta_{-}$vs. $\varphi_{c}$ since we have already known from table 2 that in average the value $\Delta \theta_{+}$will group each type of cooking oil relatively more significant than the value $\Delta \theta_{\text {- }}$. It should be noticed that we cannot only use $\theta_{\mathrm{Avg}}$ vs. $\varphi_{\mathrm{c}}$ [1], and in this method it should be modified using $\Delta \theta_{+}$(or $\Delta \theta_{-}$) vs. $\varphi_{\mathrm{c}}$ for all samples and its derivation of $\Delta \theta_{+}$vs. $\varphi_{\mathrm{c}}$ for vegetable oils. 
Second, here we propose another possibility using $\theta_{\min }$ in which $\varphi_{\mathrm{c}}$ takes place. The value $\theta$ in the range $\theta_{0} \leq \theta \leq \theta_{\min }$ is an absolute change of angle or positive angle. In the actual measurement, the change of polarization angle can be described in the left or right direction. At starting point of $\varphi=0^{\circ}$, relative to the axis of analyser, the change of polarization $\theta_{0}$ always negative sign as indicated that the direction of polarization change in the leftand the value $\theta$ approaches zero at $\varphi=\varphi_{\mathrm{c}}$. For $\varphi>\varphi_{\mathrm{c}}$ the value $\theta$ is positive and tends to maximum value at $\varphi=$ $90^{\circ}$ for all samples. And we define then that polarization angle in left and in right direction with minus and positive sign of $\theta_{\min }$, respectively. The complement model of mapping cooking oils as shown in Fig. 8 uses axis $\theta_{\min }$ and $\varphi_{\mathrm{c}}$ for all samples.

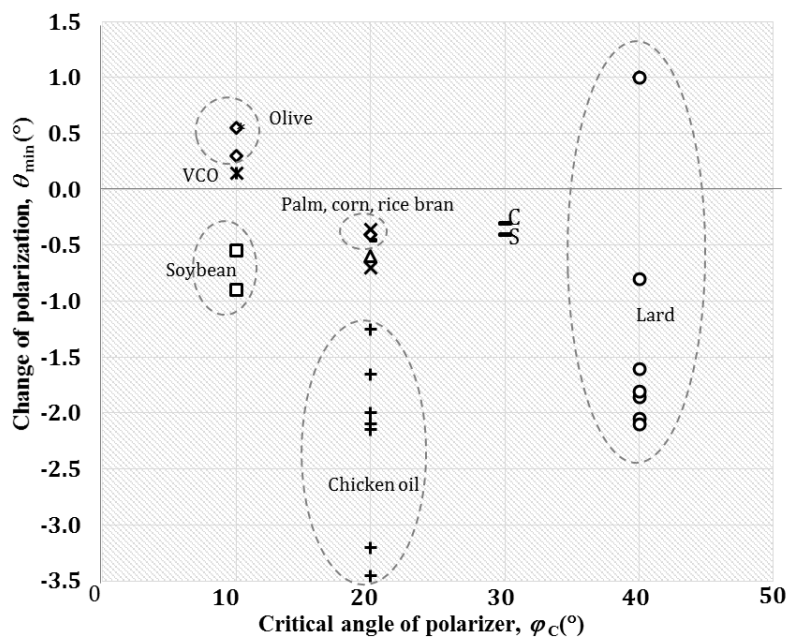

Fig. 8: Modified mapping sample using axis $\theta_{\min }$ vs. $\varphi_{c}$

Modified mapping using axis $\theta_{\min }$ and $\varphi_{\mathrm{c}}$ seems to be able to resolve olive and soybean oils significantly. The rice bran and corn look like to remain overlapped each other, and if we use restricted range $-0.8<\theta_{\min }<-0.2$ the map becomes clear as shown in fig.9.

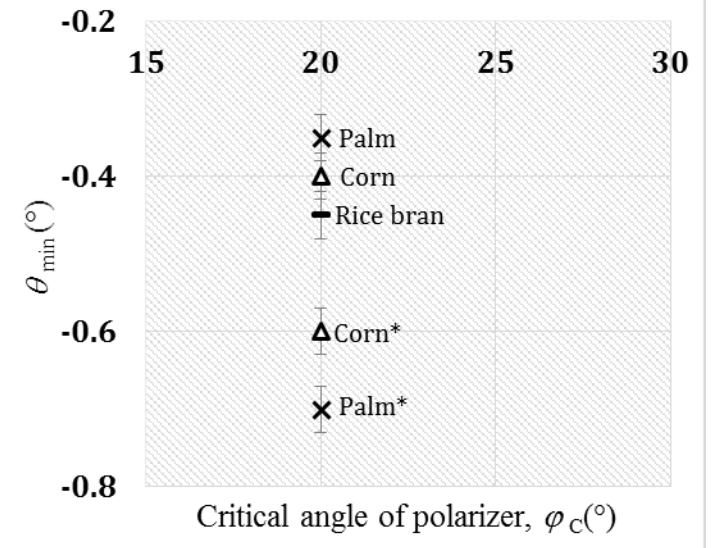

Fig. 9: Mapping using axis $\theta_{\min }$ vs. $\varphi_{\mathrm{c}}$ for rice bran and corn

Indeedwith narrow range $-0.8<\theta_{\min }<-0.2$ it still shows that rice bran and corn are overlapped each other due to the value of rice $\theta_{\min }=-0.45^{\circ} \pm 0.03^{\circ}$ and the value of corn $\theta_{\text {min }}=-0.40^{\circ} \pm 0.03^{\circ}$. As we have mentioned above that this similarity is caused by the high similarity distribution of fatty acids composition. The discrepancy of value $\theta_{\min }$ between corn and corn*, and also between palm and palm*indicates that the modified combination of the model can also be used to evaluate relatively different oil quality among the cooking oils.

The overall models described before are better than the previous results $[1,2]$, by means we can combine the modified models such as: (1) a pair of mapping using axis $\theta_{0}$ vs. $\varphi_{\mathrm{c}}$ and axis $\theta_{\min }$ vs. $\varphi_{\mathrm{c}} ;(2)$ a pair of mapping using axis $\theta_{\mathrm{p}} \mathrm{vs} . \varphi_{\mathrm{c}}$ and axis $\theta_{\min } \mathrm{vs.}$ $\varphi_{c}$; (3) a pair of modified mapping using axis $\Delta \theta$-vs. $\varphi_{c}$ and axis $\theta_{\text {min }}$ vs. $\varphi_{c}$; or (4) a pair of modified mapping using axis $\Delta \theta_{+}$vs. $\varphi_{\mathrm{c}}$ and axis $\theta_{\min }$ vs. $\varphi_{\mathrm{c}}$.The later model in average shows much better than others. This map seems capable to group various vegetable oils or animal oils, to differentiate vegetable oils from animal oils, and to develop a testing lard contamination for halal case. The mapping cooking oils in overall can be also used to determine the degradation of cooking oil quality using modified map at very narrow range of polarization angle.

The change of fluorescence polarization angle in the measurement uses combination of pure scattering and fluorescence scattering, in which the pure scattering wavelength and fluorescence wavelength are overlapped. It is a very interesting research if we can use just pure scattering or just pure fluorescence that can be overcome by using filter of wavelength.In the transmission light, the transmission wavelength is the same with the incoming wavelengthandits direction [8], and so the change of polarization is observed in relative small angle and does not depend on the polarizer's angle.By applying electro-optics effect on sample in transmission case, it can increase the change of polarization and it has provided other interesting results [9].The physical variable of observation of fluorescence polarization that is perpendicular to the incoming light is the same with transmission polarization i.e. change of polarization angle, but provides more physical properties in fluorescence phenomena.The possibilities of combination transmission electro-optics and fluorescence electrooptics have been considering and developing. It is hopeful also to provide, at least, a complement results with other spectroscopy or GCMS methods. The mapping or grouping various cooking oils through the direct measurement of fluorescence polarization angle is simpler than other sophisticated expensive spectroscopy methods or GCMS methods with their derivation's instruments. This method provides also simply understanding of physical phenomenon especially for scattering light application in cooking oils.

\section{Conclusions}

The new result of critical polarizer's angle $\varphi_{c}$ can group vegetable cooking oils as group 1 (at $\varphi_{\mathrm{c}}=10^{\circ}$ ) for VCO, olive, and soybean, as group 2 (at $\varphi_{\mathrm{c}}=20^{\circ}$ ) for palm, corn and rice bran, and as group 3 (at $\varphi_{c}=$ $30^{\circ}$ ) for sunflower and canola. Due to the various different fatty acids composition, mostly vegetable cooking oils can be distinguished using a pair of combination of the best modified map in axis $\Delta \theta_{+}$vs. $\varphi_{c}$ and axis $\theta_{\min }$ vs. $\varphi_{c}$, The choice of axis $\theta_{\min }$ vs. $\varphi_{c}$ provides not only physically explanation of 
polarization change in the left or in right direction, but also can increase the resolution using very narrow $\theta_{\min }$ to differentiate between olive and soybean oil. However, it is an exception that the similarity of corn and rice bran with an overlapped value $\theta$ cannot be resolved because ofidentical composition in fatty acids distribution. The mapping cooking oils in overall can be also used to determine the degradation of cooking oil quality using modified map at very narrow range of polarization angle. Generally, the various polarization angle $\theta$ and critical value $\varphi_{\mathrm{c}}$ can be caused by: (1) various fatty acids distribution in oil sample that can yield to a combination of small or (and)big size of triglyceride molecules and a combination of asymmetric or (and)symmetric triglyceride molecules; (2) orientation of the molecules that can effectively change of polarization of scattered fluorescence light; and (3) a complex process at transition of energy level of fluorescence polarization that the combination of pure scattering and fluorescence occurs.

The new finding of critical polarizer's angle $\varphi_{\mathrm{c}}$ can group also animals cooking oils as group 4 (at $\varphi_{\mathrm{c}}$

\section{References}

1. M. Azam, I. Afiefah, R. W. Septianti, N. K. Putri, H. Sugito, and K. S. Firdausi, "Method of fluorescence polarization for a new alternative tool for investigation of cooking oil and lard," Journal of Physics: Conference Series (to be published).

2. K. S. Firdausi, N. Simbolon, and $H$. Sugito, "Polarisasi fluoresens untuk evaluasi mutu minyak goreng,"Jurnal Ilmiaheknosains, 3, 34-39 (2017).

3. E.Sikorska, I.Khmelinskii, and M.Sikorski, Analysis of Olive Oils by Fluorescence Spectroscopy: Methods and Applications, In Tech Open 63-68 (2012).

4. Y. G. M. Kongbongan, H. Ghalila, M. B. Onana, Y. Majdi, Z. B. Lakhdar, H. Mezlini, and S. SevetreGhalila, "Characterization of Vegetable Oils by Fluorescence Spectroscopy," Food and Nutrition Sciences 2, 692-699 (2011).

5. M Saleem, Naveed Ahmad, H Ali, M Bilal, Saranjam Khan, RahatUllah, M Ahmed and S Mahmood, Investigating temperature effects on extra virgin olive oil using fluorescence spectroscopy, Laser PhysicsLetters, 27, 125602, (2017). $=20^{\circ}$ ) for chicken oil and as group 5 (at $\varphi_{\mathrm{c}}=40^{\circ}$ ) for lard. The chicken oil is distinguishable from vegetable oils due to very large value $\theta$. Lard with $\varphi_{\mathrm{c}}=$ $40^{\circ}$ is automatically distinguished from chicken oil. The large difference $\varphi_{\mathrm{c}}$ and $\theta$ of lard from vegetable oils provides an advantage to develop for testing halal oil due to lard contamination. The trace ofa test of lard contamination in minimum level detection is an important investigation to be conducted in future and developed widely for other halal food investigation. The capability of direct measurement of polarization angle for mapping various cooking oils has benefits, at least, as a complement and simple method in comparison to other expensive instruments such as IR or fluorescence spectroscopy or sophisticated GCMS methods with their derivation's instruments.

\section{Acknowledgment}

The research was supported by PTUPT and PDUPT scheme of Diponegoro University 2018.

6. C. T. Tan and S. Lock, "Are pork extracts present in my gummy bear? Gelatine speciation by LCMS/MS," AB SCIEX (2014).

7. K. S. Firdausi, H.Sugito, and N. K. Putri," Simple direct observation of polarization changes of Rayleigh scattering on sugar solution at low concentration," Journal of Physics: Conference Series, 1025, 012009 (2018).

8. K. S. Firdausi, H. Sugito, H. Rahmawati, A. B. Putranto,"The relationship between electrooptics gradient and fatty acids composition in a new investigation on palm oil quality," Advanced Science Letters,23, 6579-6581 (2017).

9. K. S. Firdausi, M. Azam, H. Sugito, R. W. Septianti, and I. Afiefah," Determination of relative dissociation energy from electro-optics as a new single-proposed parameter of vegetable oil quality,"Journal of Physics: Conference Series (to be published). 